\title{
Editorials
}

\section{Psychological and biological mechanisms of cytokine induced depression}

\author{
NILAY HEPGUL, VALERIA MONDELLI, CARMINE M. PARIANTE
}

\begin{abstract}
Depression is frequently seen in patients with medical illnesses yet the link between medical illnesses and depression remains unclear. There is increasing data to suggest that the array of depressive symptoms experienced by the medically-ill may involve inflammation. The activation of the immune system and the subsequent release of innate immune products such as cytokines can have important effects on behaviour. The treatment of choice for chronic viral hepatitis $\mathrm{C}$, interferon-alpha IFN- $\alpha$, acutely induces the production and release of other innate immune cytokines, and has been indicated to cause clinically significant depression in $30 \%$ of patients receiving treatment. This in turn can impair quality of life and affect treatment compliance. We and others use IFN- $\alpha$ induced depression as a model to identify alterations in psychological and biological pathways that predispose to depression in the medically-ill, and thus provide an explanatory link between inflammation and the subsequent behavioural changes. In this editorial, we aim to describe the main biological pathways involved in IFN-induced depression and to discuss psychological, clinical and biological factors that have been found to predict those who will develop more severe psychiatric symptoms during treatment with IFN- $\alpha$. Among these, particular attention would be given to psychological traits, genetic polymorphisms regulating inflammation and serotonergic function, and changes in plasma levels of pro-inflammatory cytokines.
\end{abstract}

Declaration of Interest: None.

Previous studies have shown that patients who are medically-ill have a significantly increased risk of developing psychiatric symptoms, such as depression or anxiety (Wise \& Taylor, 1990). Major depression is among the most frequently observed psychiatric co-morbidities seen in medical patients, and its presence is specifically associated with poor outcomes. This may be due to a reaction to the psychological stress of illness, a preexisting psychiatric disorder, a manifestation of the medical condition, or an adverse effect of medication (Wise $\&$ Taylor, 1990). The presence of depression considerably worsens medical prognosis in such patients, as it hinders treatment compliance, impairs physical and cognitive function, diminishes quality of life, increases morbidity, and in some cases can decrease survival rates (Evans et al., 2005).

Address for correspondence: Dr. C.M. Pariante, Section of Perinatal Psychiatry \& Stress, Psychiatry and Immunology, The James Black Centre, 125 Coldharbour Lane, London, SE5 9NU (United Kingdom). Fax: +44-207-848 0807

E-mail: carmine.pariante@kcl.ac.uk
Research over the last few years has shown that patients with major depression have evidence of increased inflammatory biomarkers, even not in the context of being medically-ill. An important question is whether these increased inflammatory biomarkers are "state" biomarkers that follow the development of depression, or "at-risk" biomarkers that confer vulnerability to develop depression. Strategies to understand the underlying mechanisms of depression have been mainly aimed at the general population, with major depressive patients who are otherwise physically healthy showing activated inflammatory pathways (Raison et al., 2006). Recent theories have suggested that immune factors may also contribute to the development of depression in those who are medically-ill. Studies have shown that innate immune cytokines can influence pathophysiological domains such as neurotransmitter metabolism, neuroendocrine function and regional brain activity, all of which are relevant to depression (Dantzer $e t$ al., 2008). The subsequent effects of high levels of proinflammatory cytokines on behaviour, such as depressed mood, fatigue, anxiety, sleep disturbances, anhedonia and cognitive dysfunction; closely resemble symptoms related to major depression (Capuron et al., 2002). 
Our group and others have used interferon-alpha (IFN$\alpha$ )-induced depression as a model to identify the specific alterations and activations in the immune system pathways that may be involved in instigating the behavioural changes leading to depression. IFN- $\alpha$ is a cytokine released by the innate immune system in response to viral infections, and has been shown to acutely induce the production and release of other innate immune cytokines such as interlukin-6 (IL-6) and tumour necrosis factoralpha (TNF- $\alpha$ ) (Raison et al., 2008). As well as this, IFN$\alpha$ is also thought to facilitate the recognition of virusinfected or tumour cells by cytolytic T-lymphocytes (Wichers \& Maes, 2002). As such, IFN- $\alpha$ possesses major antiviral and immunomodulatory properties. The high rate of depression during antiviral treatment with IFN- $\alpha$ is consistent with the overwhelming evidence, mentioned above, that increased inflammatory processes, activated by chronic psychosocial stress, participate in the pathogenesis of major depression (Miller, 2009). Moreover, the study of the biological mechanisms underlying IFN- $\alpha$ induced depression, may help to identify patients who are at "high risk" of developing IFN- $\alpha$ induced depression, and thus avoiding or minimising its psychiatric adverse effects. Finally, this model could also be used to clarify how pro-inflammatory processes participate in major depression, and thus identify biomarkers and drug-development targets that are clinically relevant for all patients with major depression, even outside the context of hepatitis infection.

Combined with ribavirin, IFN- $\alpha$ is the treatment of choice for patients with chronic hepatitis $\mathrm{C}$ virus (HCV) infection. This treatment, given for 24-48 weeks, clears the virus in $42-80 \%$ of cases (Agarwal et al., 2007). Unfortunately, around $30 \%$ of patients experience clinically significant depression, and up to $50 \%$ experience neuropsychiatric adverse affects (Asnis \& De La Garza, 2006). An interesting debate, yet unsolved and too complex to be discuss exhaustively in this editorial, is whether or not subjects with pre-existing depression and other psychiatric diagnoses are at higher risk of developing depression during therapy with IFN- $\alpha$ (Pariante et al., 1999; 2002). Furthermore, the experience of depressive symptoms during the course of the antiviral treatment has important negative consequences, such as impairing quality of life, reducing compliance as well as leading to dose reduction or discontinuation of treatment (Asnis \& De La Garza, 2006). All of these compromise the therapeutic response to the treatment; therefore it is extremely important, from a clinical point of view, to find out how to avoid or minimise IFN- $\alpha$ induced depression.
Prophylactic treatment with antidepressants in the form of selective serotonin reuptake inhibitors (SSRIs) has been shown to be effective in preventing IFN-induced depression in patients with malignant melanoma, who receive much higher doses of IFN-and have a much higher incidence of depression than the HCV population (Musselman et al., 2001). However, attempts of using prophylactic treatment with antidepressants in patients with chronic $\mathrm{HCV}$ infection have shown less promising results. The only two randomised placebo-controlled trial studies that have tested prophylactic treatment in these patients have failed to find major differences between placebo and antidepressants (Raison et al., 2007; Morasco et al., 2007). Moreover, some studies suggest extreme caution in the use of SSRIs in this condition. Indeed, SSRIs have antithrombotic action that further increases the risk of haemorrhages in these patients, in the presence of IFN-induced thrombocytopenia and oesophageal varices (Weinrieb et al., 2003). As well as this, patients receiving IFN are at risk of developing mania, a risk which is notably increased by antidepressants. Furthermore, the altered liver function found in these patients could change the metabolism of antidepressants, with additional toxicity risks associated with potentially higher plasma concentrations of these drugs. Finally, many patients are reluctant to take psychoactive medication, particularly given an often prolonged history of drug abuse. Therefore, prophylactic treatment "for all" is not a feasible option in these patients; consequently, it is highly important to better understand the underlying mechanisms by which IFN- $\alpha$ induced depression develops.

It is widely acknowledged that major depression, in otherwise healthy patients, occurs due to changes in the balance of neurotransmitters, in particular serotonin (5HT), as well as alterations in endocrine and immune functions. The role of abnormal serotonergic activity in depression has been highlighted by several studies, and it has been partly linked to the function of the serotonin transporter (5-HTT), known to regulate 5-HT uptake into pre-synaptic neurons. Indeed, cytokines, and in particular pro-inflammatory cytokines such as IL-6, IL-1 and TNF$\alpha$, are able to change the amount and activity of serotonin transporter and thus participate in the pathogenesis of depression. Interestingly, cytokines might also be involved in the pathogenesis of depression by acting on different biological pathways. Previous studies have shown that cytokines modulate the activity of the main biological system involved in the stress response; the hypothalamic-pituitary-adrenal (HPA) axis, which has been consistently reported to be involved in the pathogenesis of depression (Wichers et al., 2007). The pathogene-

Epidemiologia e Psichiatria Sociale, 19, 2, 2010 
sis of depression in those who are medically ill appears to be caused by similar mechanisms, and in this editorial we will describe more in detail the main hypothesised pathways through which treatment with IFN- $\alpha$ can induce depression in patients suffering from HCV infection.

In general, IFN- $\alpha$ induces changes in inflammatory biomarkers in all patients, with patients developing depression showing even larger changes in these biomarkers when compared with patients who do not develop depression (Capuron \& Miller, 2004). Specifically, treatment with IFN- $\alpha$ is associated with increased levels of a number of pro-inflammatory cytokines in the serum and cerebrospinal fluid (CSF), such as intercellular adhesion molecule-1 (ICAM-1), interleukins IL-1, IL-1 receptor antagonist (IL-1RA), IL-6, IL-8, monocyte chemoattractant protein-1 (MCP-1), TNF- $\alpha$, as well as their soluble receptors. Furthermore, those patients who develop depression show an even larger increase in some of these proinflammatory cytokines (Wichers et al., 2007; Raison et al., 2008).

In turn, the increased inflammation activates the enzyme indolamine 2,3 dioxygenase (IDO) in peripheral cells, which breaks down tryptophan; the amino acid precursor of serotonin, in its metabolites such as kynurenine, kynurenic acid, 3-hydroxykynurenine and quinolinic acid. Again, these effects are present in all patients, but patients with IFN- $\alpha$-induced depression show even lower levels of serum tryptophan and serotonin, and even higher levels of serum tryptophan metabolites (Bonaccorso et al., 2002; Wichers et al., 2005). Following IDO activation, both the reduced peripheral availability of tryptophan (putatively leading to reduced serotonin synthesis in the brain) and the production of neurotoxic tryptophan metabolites are considered essential steps in the pathophysiological processes leading to IFN- $\alpha$-induced depression (Capuron \& Miller, 2004). In fact, kynurenic acid is an N-methyl d-aspartate (NMDA) receptor antagonist, and is generally considered neuroprotective, whereas 3-hydroxykynurenine and quinolinic acid are NMDA receptor agonists which are considered neurotoxic and thus potentially contributing to the development of depression (Mynt et al., 2007). Indeed, increased proinflammatory cytokines enhance the kynurenine-3monooxygenase enzyme, which degrades the kynurenine into 3-hydroxykynurenine and thus diverts the kynurenine pathway more into neurotoxic pathway (Mynt et al., 2007). It is of note that a recent study has confirmed the relevance of this model using direct brain (CSF) investigation of inflammatory and serotonergic biomarkers in patients with IFN- $\alpha$-induced depression (Raison et al., 2008). In these patients, lower CSF levels of the sero- tonin metabolite, 5-hydroxyindoleacetic acid (5-HIAA), indicating lower brain serotonergic activity, are correlated with higher CSF levels of IL-6 and higher depressive symptoms (Raison et al., 2009). Interestingly, IDO activation has been shown also in patients with major depression not related to IFN- $\alpha$ (Mynt et al., 2007).

It is also of note that additional, non-mutually exclusive pathways by which IFN- $\alpha$ may induce depression have also been proposed. For example, increased inflammation may lead to depression also by reducing the function of the glucocorticoid receptor (GR), a process named "glucocorticoid resistance". This then leads to increased activity of the HPA axis, a consistent finding in the pathophysiology of major depression (Pariante, 2004; Pariante $\&$ Lightman, 2008). Indeed, we were the first to demonstrate that proinflammatory cytokines have the direct ability to reduce GR function, a finding later replicated by other research groups, and now conceptualised as one of the key mechanisms by which psychosocial stressors affect endocrine and immune functions (Pariante, 2004). GR function (and its modulation) can be successfully measured in vitro in peripheral blood mononuclear cells (PBMCs), as shown by us and others (Pariante, 2004). Few studies have assessed the relationship between IFN- $\alpha$ and HPA axis. In one study, IFN- $\alpha$ has been shown to induce a progressive increase in cortisol output during the day, accompanied by a reduction in the cortisol awakening response, both becoming significant after 8 weeks of treatment (Wichers et al., 2007). However, another study has found that IFN-induced depression is associated with an increase in the evening cortisol levels, and a consequent flattening of the cortisol rhythm (that is, a smaller difference between the morning peak and the evening through), an abnormality also described in major depression. Moreover, an exaggerated cortisol response to the first injection of IFN- $\alpha$ has been shown to predict the future occurrence of depression, suggesting that a "hyperreactive" HPA axis is a risk factor for developing IFN- $\alpha$ induced depression (Capuron et al., 2003). Taken together, there is therefore some evidence that the HPA axis is involved in IFN- $\alpha$-induced depression.

Some psychological, clinical and biological factors have been shown to predict the occurrence of IFN- $\alpha$ induced depression. Clinical and psychological factors including baseline depressive symptoms, neuroticism (Lotrich et al., 2007), and family history of psychiatric disorders (Asnis \& De La Garza, 2006) have also been outlined as predictors of IFN- $\alpha$-induced depression. Interestingly, both depression and neuroticism are associated with increased inflammation (Bouhuys et al., 2004) and increased awakening cortisol response (Bhagwagar

Epidemiologia e Psichiatria Sociale, 19, 2, 2010 
et al., 2005), both of which, as discussed above, are regulated by IFN- $\alpha$. Further to this, it is interesting to note the absence, among the clinical predictors that have been investigated, of a "usual suspect" risk factor for the development of depression, especially in relationship with stress and inflammation - a history of childhood trauma. There is clear evidence that childhood trauma predisposes to adult depression, an effect that is modulated by the 5-HTTLPR genetic profile (Lotrich et al., 2007). Moreover, it is well documented that a history of childhood trauma predisposes to increased inflammation in adulthood, as shown by higher levels of C-reactive protein (CRP) and fibrinogen, as well as by increased reactivity of the HPA axis (Danese et al., 2008). Finally, few studies have evaluated the link between depression and patient's health related quality of life during therapy, which can be influenced not only by the biological and symptomatic aspects of the therapy but also by pre-existing trait features such as a persons perception of their health status. The manner in which patients perceive their illness and subsequent therapy is likely to influence many aspects of their experience, including the severity of sideeffects (such as the development of depression) and health outcome (Hunt et al., 1997).

Among the biological factors, genetic factors have been described at baseline, prior to starting IFN- $\alpha$ treatment, to predict the future development of depression. We have recently found that a functional polymorphism in the promoter regions of the IL-6 gene (rs1800795) and of the serotonin transporter gene (5-HTTLPR) predict the development of IFN- $\alpha$ induced depression (Bull et al., 2009). Specifically, we have found that the "G" allele of the IL-6 gene (rs1800795) (a "risk allele" for exaggerated immune responses) increases the risk of IFN- $\alpha$ induced depression. Moreover, we have also found that the "s" allele of the serotonin transporter gene polymorphism (a "risk allele" for depression; Caspi et al., 2003) also increases the risk of IFN- $\alpha$ induced depression. More recently, we have investigated the role of N-3 (or omega-3) polyunsaturated fatty acids (PUFAs) in IFN- $\alpha$ induced depression. In fact, PUFAs play an important role in major depressive disorder (Freeman et al., 2006) as well as in cytokine induced sickness behaviour (Kozak et al., 1997). We have examined, in a Chinese sample, polymorphisms in the phospholipase A2 (PLA2) and cyclo-oxygenase 2 (COX2) genes (the two key enzymes in PUFA metabolism), together with the erythrocyte levels of the three main PUFAs, docosahexaenoic acid (DHA), eicosapentaenoic acid (EPA) and arachidonic acid (AA)(Su et al., 2009). We have found that patients who develop IFN- $\alpha$-induced depression have higher fre- quency of the PLA2 BanI GG or COX2 rs4648308 AG genotypes. Interestingly, these "at risk" genotypes are also associated with lower levels of the anti-inflammatory PUFAs, DHA and EPA, at baseline or during IFN- $\alpha$ treatment, suggesting once again that increased reactivity of the inflammatory processes (or, in this case, lack of "restrain" on the inflammatory processes by the lower PUFAs levels) is fundamental in the development of the depressive symptoms. This study is a successful example of how measuring different predictive biomarkers in the same subjects (in this case, genes variants together with their gene products) could lead to a better understanding of the molecular mechanism underlying these effects.

In summary, antiviral therapy with INF- $\alpha$ has proinflammatory effects. This pro-inflammatory response is beneficial for viral clearance, but in $30 \%$ of patients could cause clinical depression. The role of pre-existing susceptibility factors, such as psychological and genetic traits, is an important area of research currently under intense scrutiny. This knowledge will allow us to prevent the onset of the cytokine-induced depression by adequate and appropriate therapy with psychological interventions and antidepressants prior to antiviral therapy, or to target high risk subjects with very early therapeutic intervention during onset of depression.

\section{REFERENCES}

Agarwal K., Cross T.I. \& Gore C. (2007). Chronic hepatitis C. British Medical Journal 334, 54-55.

Asnis G.M. \& De La Garza (2006). Interferon induced depression in chronic hepatitis $\mathrm{C}$ : a review of its prevalence, risk factors, biology and treatment approaches. Journal of Clinical Gastroenterology 40, 322-335.

Bhagwagar Z., Hafizi S. \& Cowen P.J. (2005). Increased salivary cortisol after waking in depression. Psychopharmacology 182, 54-57.

Bonaccorso S., Marino V., Puzella A., Pasquini M., Biondi M., Artini M., Almerighi C., Meltzer H. \& Maes M. (2002). Increased depressive ratings in patients with hepatitis $\mathrm{C}$ receiving interferon-alpha-based immunotherapy are related to interferon-alpha-induced changes in the serotonergic system. Journal of Clinical Psychopharmacology 22, 86-90.

Bouhuys A.L., Flentge F., Oldehinkel A.J. \& van den Berg M.D. (2004). Potential psychosocial mechanisms linking depression to immune function in elderly subjects. Psychiatry Research 127, 237-245.

Bull S.J., Huezo-Diaz P., Binder E.B., Cubells J.F., Ranjith G., Maddock C. , Miyazaki C., Alexander N., Hotopf M., Cleare A.J., Norris S., Cassidy E., Aitchison K.J. , Miller A.H. \& Pariante C.M. (2009). Functional polymorphisms in the interleukin-6 and serotonin transporter genes, and depression and fatigue induced by interferonalpha and ribavirin treatment. Molecular Psychiatry 14, 1095-1104.

Capuron L. \& Miller A.H. (2004). Cytokines and psychopathology: lessons from interferon-alpha. Biological Psychiatry 65, 819-824.

Capuron L., Gumnick J.F., Musselman D.L., Lawson D.H., Reemsnyder A., Nemeroff C.B. \& Miller A.H. (2002). Neurobehavioural effects on interferon-alpha in cancer patients: phenomology and paroxetine responsiveness of symptom dimensions. Neuropsychopharmacology $26,643-652$. 
Capuron L., Raison C.L., Musselman D.L., Lawson D.H., Nemeroff C.B. \& Miller A.H. (2003). Association of exaggerated HPA axis response to the initial injection of interferon-alpha with development of depression during interferon-alpha therapy. American Journal of Psychiatry 160, 1342-1345.

Caspi A., Sugden K., Moffitt T.E., Taylor A., Craig I.W., H.L. Harrington, J. McClay, J. Mill, J. Martin, A. Braithwaite \& R. Poulton (2003). Influence of life stress on depression: moderation by a polymorphism in the 5-HTT gene. Science 301, 386-389.

Danese A., Moffitt T.E., Pariante C.M., Ambler A., Poulton R. \& Caspi A. (2008). Elevated inflammation levels in depressed adults with a history of childhood maltreatment. Archives of General Psychiatry 65:409-415.

Dantzer R., O'Conner J.C., Freund G.G., Johnson R.W. \& Kelley K.W. (2008). From inflammation to sickness and depression: when the immune system subjugates the brain. Nature Reviews Neuroscience 9, 46-56.

Evans D.L., Charney D.S., Lewis L., Golden R.N., Gorman J.M., Krishnan K., Nemeroff C., Bremner J., Carney R. \& Coyne J. (2005). Mood disorders in the medically ill: scientific review and recommendations. Biological Psychiatry 58, 175-189.

Freeman M.P., Hibbeln J.R., Wisner K.L., Davis J.M., Mischoulon D., Peet M., Keck P.E. Jr, Marangell L.B., Richardson A.J., Lake J. \& Stoll A.L. (2006). Omega-3 fatty acids: evidence basis for treatment and future research in psychiatry. Journal of Clinical Psychiatry 67, 1954-1967.

Hunt C.M., Dominitz J.A., Philipis-Bute B., Waters B., Blasi U. \& Williams D.M. (1997). Effect of interferon-alpha treatment of chronic hepatitis $\mathrm{C}$ on health related quality of life. Digestive Diseases and Sciences 42, 2482-2486.

Kozak W., Soszynski D., Rudolph K., Conn C.A. \& Kluger M.J. (1997). Dietary n-3 fatty acids differentially affect sickness behavior in mice during local and systemic inflammation. American Journal of Physiology 272, 1298-1307.

Lotrich F.E., Rabinovitz M., Gironda P. \& Pollock B.G. (2007). Depression following pegylated interferon-alpha: characteristics and vulnerability. Journal of Psychosomatic Research 63, 131-135.

Miller A.H. (2009). Mechanisms of cytokine-induced behavioral changes: Psychoneuroimmunology at the translational interface. Brain, Behaviour and Immunity 23, 149-158.

Morasco B.J., Rifai M.A., Loftis J.M., Indest D.W., Moles J.K. \& Hauser P. (2007). A randomized trial of paroxetine to prevent interferon-alpha-induced depression in patients with hepatitis C. Journal of Affective Disorders 103, 83-90.

Musselman D.L., Miller A.H., Porter M.R., Manatunga A., Gao F., Penna S, Pearce B.D., Landry J., Glover S., McDaniel J.S. \& Nemeroff C.B. (2001). Higher than normal plasma interleukin-6 concentrations in cancer patients with depression: preliminary findings. American Journal of Psychiatry 158, 1252-1257.

Myint A.M., O'Mahony S., Kubera M., Kim Y.K., Kenny C., KaimBasta A., Steinbusch H.W.M. \& Leonard B.E. (2007). Role of paroxetine in interferon-alpha-induced immune and behavioural changes in male Wistar rats. Journal of Psychopharmacology 21, 841-850.
Pariante C.M. (2004). Glucocorticoid receptor function in vitro in patients with major depression. Stress 7, 209-219.

Pariante C.M. \& Lightman S.L. (2008). The HPA axis in major depression: classical theories and new developments. Trends in Neuroscience 31, 464-468.

Pariante C.M., Orru M.G., Baita A., Farci M.G. \& Carpiniello B. (1999). Treatment with interferon-alpha in patients with chronic hepatitis and mood or anxiety disorders. Lancet 354, 131-132.

Pariante C.M., Landau S. \& Carpiniello B. (2002). Interferon alfainduced adverse effects in patients with a psychiatric diagnosis. New England Journal of Medicine 347, 148-149.

Raison C.L., Capuron L. \& Miller A.H. (2006). Cytokines sing the blues: inflammation and the pathogenesis of depression. Trends in Immunlogy 27, 24-31.

Raison C.L., Woolwine B.J., Demetrashvili M.F., Borisov A.S., Weinreib R., Staab J.P., Zajecka J.M., Bruno C.J., Henderson M.A., Reinus J.F., Evans D.L., Asnis G.M. \& Miller A.H. (2007). Paroxetine for prevention of depressive symptoms induced by interferon-alpha and ribavirin for hepatitis C. Alimentary Pharmacology and Therapeutics 25, 1163-1174.

Raison C.L., Borisov A.S., Woolwine B.J., Massung B., Vogt G. \& Miller A.H. (2008). Interferon- $\alpha$ effects on diurnal hypothalamicpituitary-adrenal axis activity: relationship with proinflammatory cytokines and behaviour. Molecular Psychiatry, June 2 [Epub ahead of print].

Raison C.L., Borisov A.S., Majer M., Drake D.F., Pagnoni G., Woolwine B., Vogt G., Massung B. \& Miller A. (2009). Activation of central nervous system inflammatory pathways by interferonalpha: relationship to monoamines and depression. Biological Psychiatry 65, 296-303.

Su K.P., Huang S.Y., Chiu T.H., Huang K.C., Huang C.L., Chang H.C. \& Pariante C.M. (in press). Phospholipase A2 and cyclo-oxygenase 2 genes influence the risk of interferon-alpha-induced depression by regulating polyunsaturated fatty acids levels. Biological Psychiatry [epub ahead of print].

Weinrieb R.M., Auriacombe M., Lynch K.G., Chang K.M. \& Lewis J.D. (2003). A critical review of selective serotonin reuptake inhibitor-associated bleeding: balancing the risk of treating hepatitis C-infected patients. Journal of Clinical Psychiatry 64, 15021510.

Wichers M. \& Maes M. (2002). The psychoneuroimmuno-pathophysiology of cytokine-induced depression in humans. International Journal of Neuropsychopharmacology 5, 375-388.

Wichers M.C., Koek G.H., Robaeys G., Verkerk R., Scharpé S. \& Maes, M. (2005). IDO and interferon-alpha-induced depressive symptoms: a shift in hypothesis from tryptophan depletion to neurotoxicity. Molecular Psychiatry 10, 538-544.

Wichers M.C., Kenis G., Koek G.H., Robaeys G., Nicolson N.A. \& Maes M. (2007). Interferon-alpha-induced depressive symptoms are related to changes in the cytokine network but not to cortisol. Journal of Psychosomatic Research 62, 207-214.

Wise M. G. \& Taylor S. E. (1990). Anxiety and mood disorders in medically ill patients. Journal of Clinical Psychiatry 51, 27-32. 